mungsrechts indigener Völker relativ kurz ausfällt. Erst im vierten Teil werden die vorherigen drei Teile zusammengeführt, dabei aber nicht alle der zuvor gewonnenen Erkenntnisse wieder aufgegriffen. Durch diese Darstellungsweise wird zwar eine kurze und prägnante Übersicht über die aktuellen völkerrechtlichen Entwicklungen im Bereich des Selbstbestimmungsrechts indigener Völker erschwert; dessen ungeachtet ist das Buch ein durchaus lohnender Beitrag zur Diskussion um Inhalt und Umfang des Selbstbestimmungsrechts der Völker.

Katja Göcke, Heidelberg

Susanne Gratius (ed.)

\title{
MERCOSUR y NAFTA: Instituciones y mecanismos de decisión en procesos de integración asimétricos
}

Iberoamericana, Madrid 2008. Vervuert, Frankfurt a.M. 2008. 36,00 EUR.

ISBN 978-84-8489-361-5 (Iberoamericana) / ISBN 078-3-86527-381-9 (Vervuert)

Zwei Integrationsblöcke auf dem amerikanischen Kontinent - ein Vergleich bietet sich an. Die Unterschiede zwischen dem Gemeinsamen Markt des Südens (MERCOSUR) und der nordamerikanischen Freihandelszone (NAFTA) sind sehr ausgeprägt und könnten kaum größer sein, doch gibt es auch eine Gemeinsamkeit: die Dominanz einer starken Führungsmacht, hier Brasilien, dort die Vereinigten Staaten. Diesen asymmetrischen Integrationsbeziehungen ist der vorliegende Band gewidmet, der aus einem von der Thyssen-Stiftung geförderten und der Herausgeberin geleiteten Forschungsprojekt hervorgegangen ist, das in den Jahren 2001-2004 am damaligen Institut für Iberoamerika-Kunde in Hamburg durchgeführt wurde (für die vorliegende Publikation wurden einige Aktualisierungen vorgenommen). An dem Projekt haben Mitarbeiter des Instituts und externe Autoren mitgewirkt, doch sind Konzeption und Inhalt des Bandes deutlich von der Herausgeberin geprägt. Sie selbst hat neben der Einführung zwei der fünf Kapitel verfasst und ist außerdem an zwei der anderen maßgeblich beteiligt. Durch die Einbeziehung externen Sachverstandes (auch durch einen in der Schlussphase des Projekts veranstalteten größeren workshop) und intensive Feldforschung in den beteiligten Staaten (170 Interviews) sind vielfältige Aspekte in das Werk eingegangen. Der klare Aufbau und flüssige Duktus des Textes machen den Band zu einer anregenden und spannenden Lektüre.

In der Einführung werden beide Integrationsblöcke zutreffend in den Zusammenhang des „regionalismo abierto“ gestellt und die bestehenden Unterschiede in großen Linien skizziert. Kennzeichnend für die NAFTA, die einerseits durch die Einbeziehung des Arbeits- und Umweltsektors über die ursprünglich intendierte Freihandelszone hinausgeht, anderseits als Verbindung von zwei bilateralen Beziehungen der USA mit seinen Nachbarn Kanada und Mexiko hinter dem Modell einer einheitlichen Freihandelszone zurückbleibt, ist die ausführliche und feststehende rechtliche Regelung der Gründungsverträge. Demge- 
genüber wird der MERCOSUR, der entgegen seinem Namen erst das Stadium einer unvollkommenen Zollunion erreicht hat, geprägt durch seine von den beteiligten Regierungen gesteuerte und den politischen Einflüssen unterworfene Entwicklung, die ihren Ausdruck in der Bedeutung des Sekundärrechts findet. Die anschließende Erörterung der verschiedenen Integrationstheorien (Realismus, Neorealismus, Funktionalismus, Neofunktionalismus, Konstruktivismus, Institutionalismus, Neoinstitutionalismus), die im 1. Kapitel ihre Fortsetzung findet, vermag dieser klaren Aussage wenig hinzuzufügen. Im übrigen enthält dieses Kapitel vor allem eine Beschreibung der Institutionen und Entscheidungsprozesse in beiden Integrationsblöcken. Im MERCOSUR sind die Organe mit Entscheidungsgewalt (Rat, Gruppe und Handelskommission) intergouvernemental zusammengesetzt und von den Regierungen gesteuert, was die Umsetzung ihrer Beschlüsse in den beteiligten Staaten erleichtern soll. Im Buch wird aber auch die gleichwohl unzureichende Umsetzung dieser Beschlüsse thematisiert, wobei allerdings nicht genau zwischen der Rechtssetzung durch Staatsverträge und durch Organakte unterschieden wird, was das Verständnis erschwert (wozu auch die falsche Zählung der Fußnoten auf S. 48-55 beiträgt). Auch der Vorschlag, hier Abhilfe durch ein „fast track“-Verfahren zu schaffen, beruht auf einem Irrtum: Dieses aus der nordamerikanischen Praxis bekannte Verfahren bezieht sich allein auf Staatsverträge und lässt dem US-Kongress nur die Wahl zwischen Ablehnung und Zustimmung (ohne Änderungsmöglichkeit), was aber der Rechtslage in den MERCOSUR-Staaten ohnehin entspricht. Eine völlig andere Struktur weist das institutionelle Gefüge der NAFTA auf, das in den folgenden Abschnitten des Kapitels ausführlich (teilweise überlappend und auch widersprüchlich) geschildert wird. Die Freihandelskommission, gebildet aus den drei Handelsministern, hat wenig zu entscheiden und weder einen festen Sitz noch einen festen Tagungsrhythmus. Das Sekretariat der NAFTA besteht aus drei separaten nationalen Sektionen, die jeweils mit der Behandlung von Beschwerden gegen ihr Land befasst und unterschiedlich ausgestaltet sind (in Kanada als autonome Behörde, in den USA und Mexiko als Abteilung des Handels- oder Wirtschaftsministeriums), aber gleichwohl in gewissem Umfang miteinander kooperieren. Ansätze zu einer echten länderübergreifenden Zusammenarbeit finden sich jedoch eher in den fachlichen Arbeitsgruppen und Ausschüssen im Rahmen der Freihandelskommission sowie in den ständigen Kommissionen der Parallelabkommen für Arbeits- und Umweltfragen. Insgesamt wird das schwach ausgeprägte institutionelle System der NAFTA als praktisch erfolgreich, aber unübersichtlich und intransparent gekennzeichnet. In einem Vergleich mit dem MERCOSUR wird vor allem hervorgehoben, dass die asymmetrischen Beziehungen in beiden Integrationsblöcken sich nicht in den offiziellen Institutionen widerspiegeln, die daraus folgenden Probleme vielmehr auf politischem Wege durch bilaterale Verhandlungen oder direkte Einflussnahme der jeweils dominierenden Macht gelöst werden.

Das bei weitem umfangreichste 2. Kapitel ist der Streitschlichtung innerhalb der Integrationsblöcke gewidmet. In einem kurzen Abriss, der nur für den bereits kundigen Leser wirklich verständlich ist, werden die verschiedenen Formen der Konfliktlösung im MERCOSUR und ihre praktische Bedeutung dargestellt: direkte Verhandlungen, Be- 
schwerde- und Konsultationsverfahren vor den MERCOSUR-Organen, ad hoc Schiedsverfahren. Während im ersten Jahrzehnt ausschließlich die mehr informellen Verfahren zur Konfliktlösung benutzt wurden und vielfach zu einem dem geltenden MERCOSUR-Recht widersprechenden Ergebnis führten, ist seither eine stärkere Hinwendung zu rechtlichen Lösungen durch Schiedsverfahren festzustellen (aber nicht erst, wie der Text insinuiert, seit dem Schiedsreglement von 1998, vielmehr gab umgekehrt das erste Schiedsverfahren Anlass zu dessen Ausarbeitung). Ein separater Nachtrag informiert über die Änderungen durch das Protokoll von Olivos von 2002 (in Kraft seit 2004), erfasst aber nicht mehr die unter seiner Geltung durchgeführten Schieds- und Vorlageverfahren vor dem neuen MERCOSUR-Revisionsgericht. Wesentlich ausführlicher und anschaulicher ist die anschließende Darstellung der Mechanismen der Streiterledigung innerhalb der NAFTA, die aus drei völlig verschiedenen Verfahren bestehen: den Schiedsverfahren in Investitionsstreitigkeiten nach Cap. XI, der Einberufung binationaler Panels in Dumping-Sachen nach Cap. XIX, schließlich dem allgemeinen Verfahren vor der Freihandelskommission und den in diesem Verfahren gebildeten Panels über die Anwendung und Auslegung des Vertrages nach Cap. XX (daneben noch die Streitschlichtungsverfahren der Parallelabkommen). Insoweit besteht eine gewisse Überschneidung mit dem 1. Kapitel, wo diese Verfahren ebenfalls bereits erörtert wurden. Eine Übersicht über die Praxis ergibt, dass die Verfahren nach Cap. XIX den größten Anteil ausmachen und überwiegend zu befriedigenden Ergebnissen führen, während die allgemeinen Verfahren nach Cap. XX nur unzureichend funktionieren. Kritisiert wird hier vor allem die fehlende Verbindlichkeit und Durchsetzbarkeit der Panelbeschlüsse, was aber gegen den Widerstand der USA nicht zu ändern ist. In die Kritik geraten sind auch die Schiedsverfahren nach Cap. XI, die nach Ansicht von Umweltgruppen zu einem Vorrang von Investitionsinteressen vor Umweltbelangen führen. Der abschließende Vergleich zwischen den Streitmechanismen von NAFTA und MERCOSUR ermüdet durch viele Wiederholungen. Beide werden unter verschiedenen Gesichtspunkten einander gegenübergestellt, wobei in der Tendenz die Praxis der NAFTA als effektiver angesehen wird. Deren Verfahren stützen sich auf das feste Regelwerk der Verträge, was freilich gebotene Reformen erschwert, während sich im MERCOSUR erst eine allmähliche Entwicklung zu einer stärkeren Legalisierung der Konfliktbewältigung abzeichnet. Insbesondere das fachliche Expertenwissen der NAFTA-Panels mit ihren konkreten Lösungen kontrastiert mit den allgemein gehaltenen Rechtsausführungen der MERCOSUR-Schiedssprüche, die vielfach in der Praxis ohne nachhaltige Wirkung bleiben. In beiden Blöcken finden sich die dominierenden Mächte Brasilien und USA nicht nur besonders häufig in der Rolle des Beklagten, sondern werden auch am ehesten für die Nichteinhaltung der aus den Verfahren resultierenden Verpflichtungen verantwortlich gemacht. Die in diesen Fällen zulässige Retorsion ist für die kleineren Staaten kein geeignetes Mittel zur Durchsetzung ihrer Interessen.

Eben dieses Ungleichgewicht zwischen den jeweiligen Partnern der Integration ist Gegenstand des 3. Kapitels. Dabei wird ein sich wandelndes Bild der politischen Entwicklung in den betreffenden Ländern und den gegenseitigen Beziehungen gezeichnet, das 
keine statische Festlegung auf einfache Relationen erlaubt. Für den MERCOSUR wird gezeigt, wie Brasilien seine Führungsrolle erst unter dem Präsidenten Lula akzeptierte, der damit auch politisch-strategische Ziele im Sinne der Stärkung einer gemeinsamen Verhandlungsposition verband und das bisherige „Südprojekt“ gegenüber Venezuela öffnete. Trotz des brasilianischen Übergewichts erscheint die Bilanz für die übrigen Länder positiv. Für Argentinien gilt dies einerseits aufgrund seiner starken wirtschaftlichen Ausrichtung auf Brasilien, anderseits durch die bestehende bilaterale Achse Argentinien-Brasilien, welche die Berücksichtigung seiner Interessen sichert. Die kleineren Länder Paraguay und Uruguay haben dagegen aufgrund ihrer unterschiedlichen Entwicklung nicht zu einer gemeinsamen Haltung gegenüber den größeren Partnern gefunden. Trotz ihrer schwachen Position profitieren sie einerseits durch ihre starke wirtschaftliche Verflechtung mit diesen Ländern, anderseits durch ihre gleichberechtigte Vertretung in den MERCOSUR-Organen. Dem wird die Entwicklung der NAFTA gegenübergestellt, die durch die deutliche Hegemonie der USA und die starke Abhängigkeit Mexikos und Kanadas gekennzeichnet ist. Der politische Entscheidungsprozess für die NAFTA und deren unterschiedliche Wahrnehmung durch die verschiedenen Interessengruppen wird für alle drei Staaten in einer sehr differenzierten Sicht beschrieben, die fast jede Feststellung mit einem ,aber“ kontrastiert. In ökonomischer Hinsicht gilt die NAFTA als Erfolg, doch sind die schwächeren Gruppen davon ausgeschlossen. Zwar profitiert Mexiko von einer positiven Handelsbilanz, doch sind Ursache dafür die in Mexiko ansässigen US-Unternehmen. Mit der wirtschaftlichen Abhängigkeit wächst in Mexiko und Kanada die Sorge um die nationale Identität, doch finden auch diese Länder wegen ihrer unterschiedlichen Position nicht zu einer gemeinsamen Haltung gegenüber den USA. Besondere Konfliktpunkte zwischen den USA und Mexiko sind schließlich das ungelöste Migrationsproblem und die negative Auswirkung der NAFTA auf den mexikanischen Agrarsektor, die sich in einem Teufelskreis gegenseitig verstärken, indem die subventionierten US-amerikanischen Agrobusiness-Firmen gerade von den illegalen mexikanischen Arbeitern profitieren und durch ihre billigen Exporte den Migrationsdruck in der mexikanischen Landwirtschaft erhöhen. Das anschließende 4. Kapitel vertieft diesen Aspekt der sozialen und ökonomischen Spannungen und beschäftigt sich in allgemeiner Form mit den bestehenden Ungleichheiten innerhalb der Integrationsblöcke. Solche Ungleichheiten bestehen nicht nur im Verhältnis der beteiligten Staaten zueinander, sondern ebenso zwischen den verschiedenen Wirtschaftssektoren, den unterschiedlich entwickelten Regionen und den durch große Einkommensunterschiede getrennten sozialen Schichten. Ausgehend von der Feststellung, dass der Erfolg der Integration nicht nur am makroökomischen Wachstum, sondern ebenso an ihrer Wirkung für die Entwicklung der beteiligten Länder und der gleichmäßigen Verteilung des ökonomischen Nutzens zu messen ist, werden dafür geeignete Instrumente wie Entwicklungsbanken oder Strukturfonds erörtert, die aber wegen der fehlenden zentralen Steuerung in diesem Kontext nicht die gleiche Rolle spielen können wie etwa in der Europäischen Union.

In ihrem zusammenfassenden Ausblick erörtert die Herausgeberin im 5. Kapitel die Möglichkeiten und Grenzen einer verstärkten Integration in NAFTA und MERCOSUR. 
Hier werden noch einmal die Unterschiede und Gemeinsamkeiten beider Integrationsvorhaben herausgestellt und verschiedene Szenarien für ihre weitere Entwicklung entworfen, aber auch Vorschläge zur Lösung der strukturellen Probleme unterbreitet. Wie alle Prognosen sind die hier gezeichneten Perspektiven abhängig von einer Fülle unwägbarer Faktoren, so dass das Ergebnis letztlich offen bleibt. Insgesamt liefert das vorliegende Buch mit seiner Vielfalt an Material und Gedanken einen wichtigen Beitrag zur aktuellen Integrationsdiskussion auf dem amerikanischen Kontinent.

Jürgen Samtleben, Hamburg

\section{Thomas Jäger (Hrsg.)}

\section{Die Komplexität der Kriege}

VS Verlag für Sozialwissenschaften, Wiesbaden 2010, 307 S., 39,95 EUR

ISBN 978-3-531-17311-5

Die längste Zeit definierte sich der Krieg über seine Akteure, Staaten oder Staatengruppen. Gegenwärtig scheinen wir auf dem Rückweg in die Barbarei. Der Krieg definiert sich wieder über seine Dimensionen: Maximierung von Verheerung und entgrenzte Grausamkeit. Anders wären wir kaum Zeugen der Notwendigkeit, die Art und Weise, wie sich in Afghanistan die friedensstörenden Kräfte dem ISAF-Mandat nach Kap. VII der UN-Charta widersetzen, zumindest als "kriegsähnliche" Zustände zu bezeichnen; dies freilich nicht im Sinne mancher Begriffsverwirrung, die das ISAF-Mandat mit den Eroberungskriegen früherer Zeiten in eine Reihe stellt oder gleich vom "NATO-Angriffskrieg in Afghanistan" deliriert.

Der vorliegende Sammelband will, wie Herausgeber Thomas Jäger im Vorwort betont, den Ursachen und neuen Rahmenbedingungen, unter denen nach dem Ost-West-Konflikt der Krieg sein Gesicht - wieder einmal - gewandelt hat, nachspüren und mit seinen Analysen "... dazu beitragen, diese Komplexität weiter zu vermessen". Es geht wohlweislich nicht um die Komplexität "des Krieges". Der stattdessen verwendete Plural ist Programm. Dafür liefert gleich zu Beginn der Kölner Politologe Rasmus Beckmann einen eindrucksvollen Beleg ("Kriegstheoretische Analyse von neuen Gewaltformen"). Niemand Geringerer als Clausewitz wird hier befragt zur Katalogisierbarkeit moderner Gewaltformen. Obwohl zeitgebunden, liefert "Vom Kriege" auch heute noch Parameter zur analytischen Bewältigung der nachgerade beängstigenden Vielfalt nationaler wie internationaler Konfliktszenarien.

Diesem Auftakt folgen in drei Abschnitten verschiedene Betrachtungsweisen moderner Kriegsformen. Den ersten Abschnitt ("Kriege im Zeichen von Ordnung und Sicherheit") eröffnet Andreas Herberg-Rothe, Privatdozent am sozialwissenschaftlichen Institut der Berliner Humboldt-Universität, mit seinen "Reflexionen über den Begriff des Weltordnungskonfliktes". Sprachschöpfungen wie diese markieren den Mut machenden Weg zum 